\title{
Comparison of the effectiveness and safety of travoprost and latanoprost for the management of open-angle glaucoma given as an evening dose
}

\author{
JING LI, XIAOYI WANG, GUIHUA XU, RUIDONG DENG, \\ LAIWEI WU, LIQIN ZHANG and ZILIN CHEN \\ Department of Ophthalmology, Huizhou Municipal Central Hospital, \\ Huizhou, Guangdong 516001, P.R. China
}

Received December 10, 2019; Accepted June 5, 2020

DOI: $10.3892 /$ etm.2020.9152

\begin{abstract}
As intraocular pressure (IOP) is primarily higher in the morning, an evening dose of prostaglandin analogs is typically used as monotherapy to decrease IOP in patients with open-angle glaucoma. Travoprost (TV) has reported efficacy in treating open-angle glaucoma; however, the safety and efficacy may be different compared with that for latanoprost (LT). The aim of the present study was to compare the effectiveness and safety of an evening dose of TV compared with that of LT in treating open-angle glaucoma. Data including IOP, results of lid and slit-lamp examination and ophthalmoscopy, as well as adverse effects in 250 affected eyes from patients with open-angle glaucoma who received either TV $(n=89)$ or LT $(n=161)$ once in the evening for 3 -months were included in the analyses. At the end of treatment, TV $(23.45 \pm 1.52$ vs. $19.15 \pm 1.01 \mathrm{mmHg} ; \mathrm{P}<0.0001)$ and LT $(23.93 \pm 2.11$ vs. $19.45 \pm 1.11 \mathrm{mmHg}$; $\mathrm{P}<0.0001)$ successfully lowered the IOP. In addition, there was no significant difference in the reduction of IOP values at the end of treatment between the two groups $(\mathrm{P}=0.120)$. Furthermore, there were no adverse effects on visual acuity $(\mathrm{P}>0.05)$, except for non-visual acuity, for example hyperemia $(\mathrm{P}<0.0001$ for both groups), while there was a significant increase in the number of patients with dry eyes receiving TV $(\mathrm{P}=0.020)$ and a significant increase with eyelid swelling $(\mathrm{P}=0.036)$ and headache $(\mathrm{P}=0.037)$ in patients receiving $\mathrm{LT}$. In conclusion, evening doses of TV and LT had the same efficacy and
\end{abstract}

Correspondence to: Dr Zilin Chen, Department of Ophthalmology, Huizhou Municipal Central Hospital, 41 Eling Road, Huizhou, Guangdong 516001, P.R. China

E-mail: boydongdong1@126.com

Abbreviations: IOP, intraocular pressure; LT, latanoprost; TV, travoprost

Key words: evening dosing, hyperemia, intraocular pressure, latanoprost, open-angle glaucoma, travoprost manageable adverse effects in the treatment of open-angle glaucoma (level of evidence, 3 ).

\section{Introduction}

The death of retinal ganglion cell axons and the alteration of the optic nerve may cause optic neuropathy (1). Chronic neuropathy is termed glaucoma (2), which may lead to progressive neuropathy and then blindness (1).

The etiology of blindness varies among different populations. In developed countries, age-associated macular degeneration is the primary cause; however, in certain undeveloped countries, infections (i.e. trachoma) are the leading cause depend on/vary among ethnicities (3). The leading cause of blindness among white individuals was reported to be age-associated macular degeneration (54.4\% of cases), while among black individuals, cataract and glaucoma accounted for $>60 \%$ of cases of blindness (4). The prevalence rates and the clinical burden (e.g. glaucomatous damage, vision loss, blindness, social withdrawal due to blindness) of open-angle glaucoma are high in the Chinese population (5).

High intraocular pressure (IOP) is the major risk parameter used to determine the prevalence and development of glaucoma. Therefore, lowering the IOP is beneficial in the development of glaucoma (6). Several methods are available for reducing IOP, for example, laser treatment, surgery, and pharmaceutical treatments; however, laser treatment and surgery are less effective compared with medication (1).

An evening dose of prostaglandin analogs is typically used as monotherapy to decrease IOP (7). Latanoprost (LT) is a well-established monotherapy to treat open-angle glaucoma, initially for 2 years (8). Travoprost (TV) is a prostaglandin F2a analog (9) and has reported efficacy in open-angle glaucoma (10); however, the efficacy and safety of TV may be different compared with that for LT in treating open-angle glaucoma $(2,11)$. Certain cross-sectional studies have reported superiority of TV over LT $(7,12)$; however, other studies have reported equal efficacy and safety $(13,14)$ in the management of open-angle glaucoma. There is also a lack of available comprehensive data on the effectiveness of 
TV and associated adverse effects. Thus, further research is required.

The objectives of the present retrospective study were to compare the effectiveness and safety of an evening dose of TV compared with that for LT in the management of open-angle glaucoma.

\section{Materials and methods}

Study population. The records of patients with primary open-angle glaucoma and an IOP of $>21 \mathrm{mmHg}$ were extracted from the outpatient records from the Huizhou Municipal Central Hospital (Guangdong, China) from 1st January 2018 to 1st July 2019, and the data of 360 affected eyes were retrieved. Patients who were treated with drugs other than TV or LT (99 affected eyes) and subjected to laser therapy (11 affected eyes) were excluded from the analysis. The data on the 250 remaining patients with primary open-angle glaucoma and treated with either TV or LT [based on the decision of the ophthalmologist(s)] were retrospectively included in the analyses (Fig. 1). If patients were affected bilaterally, the patients were instructed to use drops in both eyes and the data of both eyes were included in the analysis.

Cohort. A total of 89 patients with (an) affected eye(s) were treated with $40 \mu \mathrm{g} / \mathrm{ml}$ TV eye drops (Travatan ${ }^{\circledR}$; Alcon Laboratories Inc.) once in the evening (8 pm) (15) for 3 -months and 161 patients were treated with $50 \mu \mathrm{g} / \mathrm{ml}$ LT eye drops (Xalatan; Pfizer Inc.) once in the evening (8 pm) (16) for 3-months. These two groups were termed the TV and LT group, respectively.

IOP measurements. A total of 3 different measurements were performed by ophthalmologists (with a minimum of 3-years of experience) using the Goldman Applanation Tonometer (Haag-Streit AG) in the morning (10 am), afternoon (2 pm) and evening $(6 \mathrm{pm})$ and the mean of all three measurements were used for the analysis of IOP (17).

Lid and slit-lamp examination. The lid and slit-lamp examination was performed by ophthalmologists (with a minimum of 3-years of experience). The upper and lower eyelids, the margin between the upper and the lower eyelids and any abnormal signs within the eyelids, conjunctiva, cornea, anterior chamber and the lens were examined. The abnormalities were scored using the following grading system: 0, Absent; 1, mild; 2, moderate; and 3, severe (maximum possible) (3). Conjunctival hyperemia was assessed in the temporal peripheral, nasal peripheral, central inferonasal, central superonasal, central inferotemporal and central superotemporal region of the eyes using the following classification: 0 , Absent; 1 , mild hyperemia; 2 , moderate hyperemia; and 3 , severe hyperemia (maximum possible) (Fig. 2). Conjunctival staining was evaluated in the same area using Lissamine green dye and scored as follows: 0, Absent; 1, mild staining; 2, moderate staining; and 3 , severe staining (maximum possible). Corneal staining was assessed from the superior, inferior, central, temporal and nasal area of the cornea as follows: 0 , Absent; 1 , mild staining; 2 , moderate staining; and 3 , severe staining (maximum possible; Fig. 3) (13).
Ophthalmoscopy. Ophthalmoscopy was performed by ophthalmologists (with a minimum of 3 -years of experience) to examine the optic nerve head, the vertical cup to disc ratio and the optic disc hemorrhages (3). All of the parameters were evaluated at the start of treatment during a routine check-up and at the end of treatment. The other adverse effects occurring were recorded at the end of the 3 months and analyzed.

Statistical analysis. SPSS statistics version 26 (IBM Corp.) was used for statistical analysis. Fischer's exact test was performed for ordinal and continuous data and the Mann-Whitney U test (between-groups) or the Wilcoxon test (within-group) (3) was performed for continuous variables. $\mathrm{P}<0.05$ was considered to indicate a statistically significant difference.

\section{Results}

Study population. Patients from 37 to 73 years were included in analyses. Among 250 patients, 125 patients were male and 125 patients were female $(1: 1)$. There was no significant difference in the demographical characteristics and clinical conditions between the two cohorts at the time of admission (all $\mathrm{P}>0.05$ ). The demographic and clinicopathological characteristics of the patients enrolled are provided in Table I.

Efficacy. Following 3 months of treatment, a significant reduction in IOP as compared with that at the start of treatment was obtained with TV $(23.45 \pm 1.52 \mathrm{mmHg}$ vs. $19.15 \pm 1.01 \mathrm{mmHg}$; $\mathrm{P}<0.0001)$ and LT $(23.93 \pm 2.11 \mathrm{mmHg}$ vs. $19.45 \pm 1.11 \mathrm{mmHg}$; $\mathrm{P}<0.0001)$. However, there were no significant differences in IOP values at the end of treatment between the two groups (19.15 $\pm 1.01 \mathrm{mmHg}$ vs. $19.45 \pm 1.11 \mathrm{mmHg}$; $\mathrm{P}=0.120$; Fig. 4).

Safety. Corrected visual acuity $(\mathrm{P}=0.936)$, visual field defect $(\mathrm{P}=0.367)$, and vertical cup to disc ratio $(\mathrm{P}=0.086)$ had no differences between the two groups at baseline. Following treatment for 3 months and a comparison between 3 months and the baseline, no adverse effects on visual acuity, visual field and the vertical cup to disc ratio were observed for TV and LT and the data were very similar between the two cohorts at 3 months (all P>0.05; Table II). There was also no significant difference in the qualitative slit-lamp characteristics (all P>0.05; Table III), but non-slit-lamp characteristics, for example hyperemia, were significantly increased in each group after treatment $(\mathrm{P}<0.0001$ for both drugs; Fig. 5) but that there was no significant inter-group difference. In addition, there was no significant difference in corneal staining $(0.81 \pm 0.05$ vs. $0.83 \pm 0.10 ; \mathrm{P}=0.078$; Fig. 6$)$ and conjunctival staining ( $3.11 \pm 0.08$ vs. $3.08 \pm 0.15 ; \mathrm{P}=0.081$; Fig. 7) between the two groups at the end of treatment.

Treatment-emergent adverse effects. Discomfort and irritation in the eyes were commonly reported by patients during the 3 months of treatment. There were significantly more patients with dry eyes $(\mathrm{P}=0.020)$ who received $\mathrm{TV}$ treatment, while patients treated with LT had significantly more frequent eyelid swelling $(\mathrm{P}=0.036)$ and complaints of headache $(\mathrm{P}=0.037)$ following 3 months of treatment (Table IV). 


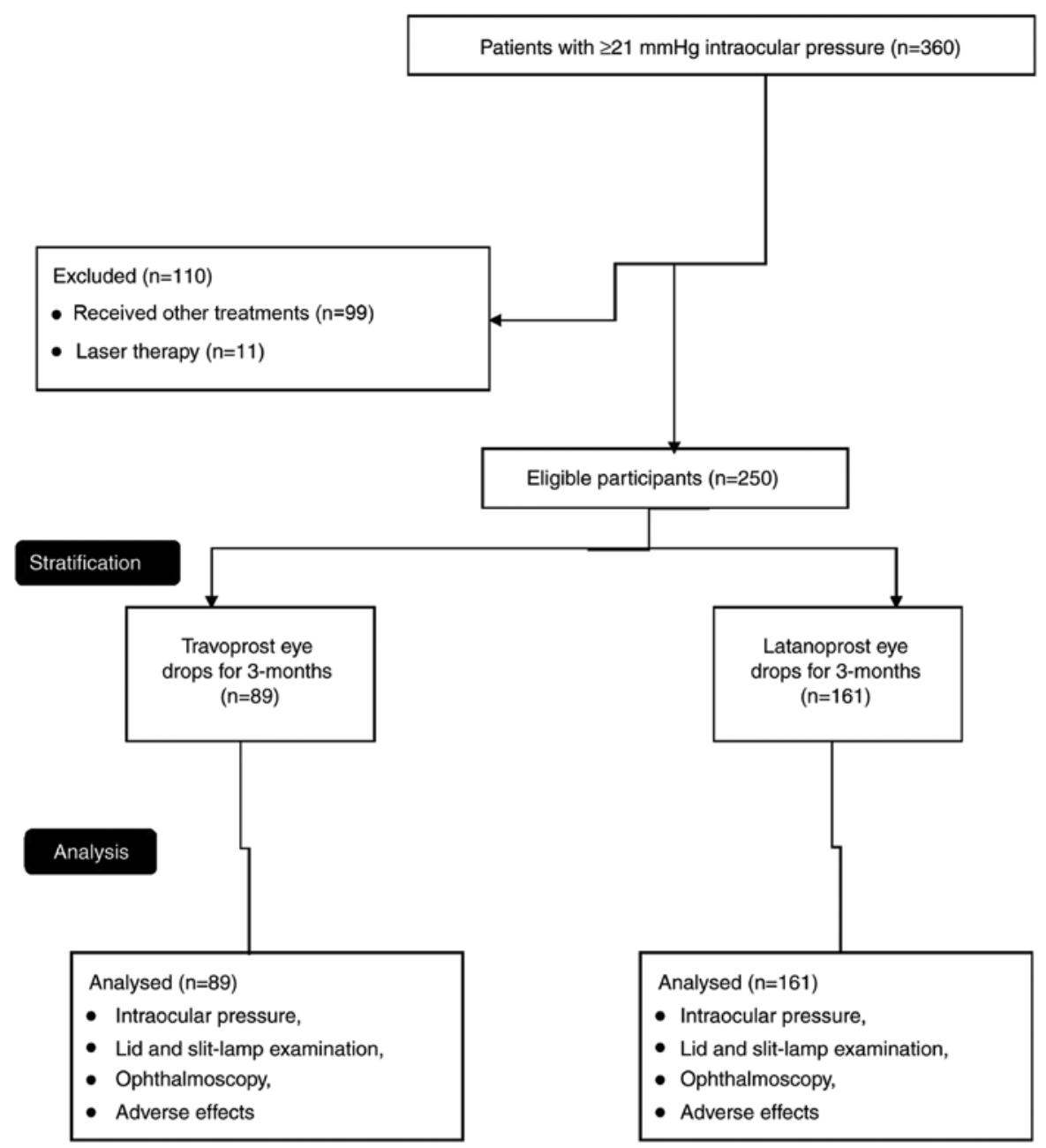

Figure 1. Flow chart of the present study.

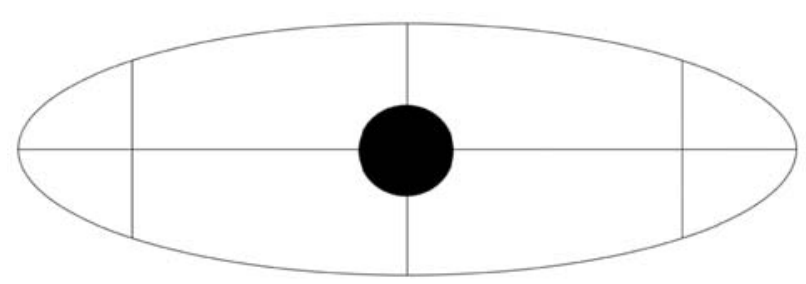

Figure 2. Evaluation of hyperemia and conjunctival staining in6areas of the eye.

\section{Discussion}

The present retrospective study indicated that the evening doses of TV and LT controlled IOP at a similar level with no significant inter-group difference $(\mathrm{P}=0.120)$ following 3 months of treatment in patients with glaucoma. The results of IOP of the present study were consistent with those of previous open-label, prospective, non-randomized cohort studies $(13,18)$, prospective randomized clinical trials (19), a single-center, open-label trial (14), a randomized crossover study (20) and a prospective observational study (21); however, they were not in agreement with those of prospective, cross-sectional studies $(7,17)$. Comparisons over time are difficult to evaluate in a cross-sectional study (22), as carry-over

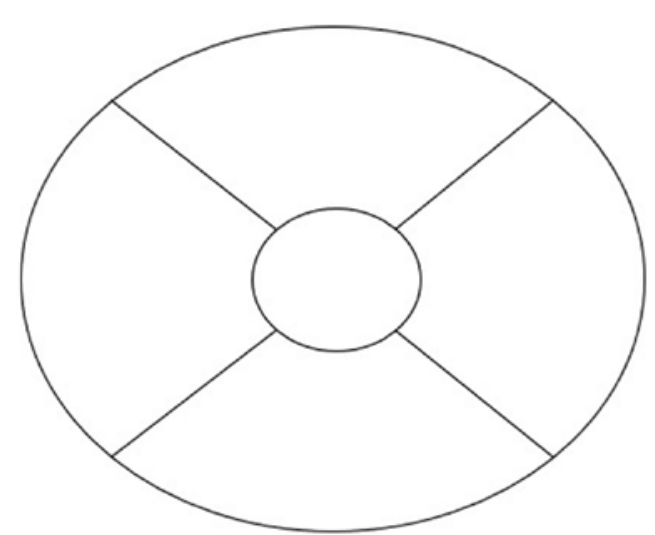

Figure 3. Evaluation of corneal staining in5areas of the cornea.

effects maybe reported in a cross-sectional study $(3,17)$, which may be a possible reason for this discrepancy. The results of the present study are also in contrast to those of a cross-over randomized trial using morning doses of TV, as the IOP is higher in the morning compared with that in the evening (higher baseline untreated IOP) (23). The present study identified that TV efficiently reduced IOP, and the efficacy was similar to that of LT. 
Table I. Demographic and clinicopathological and characteristics of the patients on admission.

\begin{tabular}{|c|c|c|c|}
\hline Characteristic & TV group $(n=89)$ & LT group $(\mathrm{n}=161)$ & P-value \\
\hline Intraocular pressure (mmHg) & $23.45 \pm 1.52$ & $23.93 \pm 2.11$ & 0.060 \\
\hline Age (years) & & & 0.459 \\
\hline Range & $37-72$ & $41-73$ & \\
\hline Mean & $63.12 \pm 11.13$ & $64.15 \pm 10.14$ & \\
\hline Sex & & & 0.792 \\
\hline Male & $43(48)$ & $82(51)$ & \\
\hline Female & $46(52)$ & 79 (49) & \\
\hline History of visual complications (months) & $40.12 \pm 5.45$ & $41.23 \pm 4.55$ & 0.087 \\
\hline Ethnicity & & & 0.910 \\
\hline Han Chinese & $80(90)$ & $147(91)$ & \\
\hline Mongolian & $8(9)$ & $12(8)$ & \\
\hline Tibetan & $1 \quad(1)$ & $2(1)$ & \\
\hline Diabetes & $11(12)$ & $18(11)$ & 0.838 \\
\hline Dyslipidemia & $8(9)$ & $18(11)$ & 0.669 \\
\hline Hypertension & 8 (9) & $13(8)$ & 0.815 \\
\hline Migraine & $15(17)$ & $25(16)$ & 0.857 \\
\hline Smoking & 7 (19) & 5 (9) & 0.122 \\
\hline Family history of glaucoma & $5(6)$ & 11 (7) & 0.793 \\
\hline
\end{tabular}

Values are expressed as the mean \pm standard deviation or $\mathrm{n}(\%)$. Fischer's exact test was performed for ordinal data and the Mann-Whitney $\mathrm{U}$ test was performed for numerical data. $\mathrm{n}$ indicates the number of eyes, for co-morbidity $\mathrm{n}$ does not indicate for eyes. TV, travoprost; LT, latanoprost.

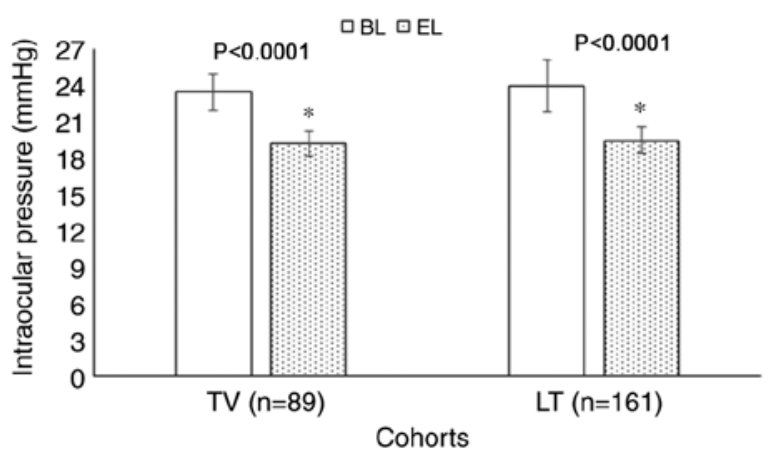

Figure 4. Intraocular pressure in the two groups at different time-points. Values are expressed as the mean \pm standard deviation. ${ }^{*} \mathrm{P}<0.05$ vs. BL. The mean of all three measurements [morning (10 am), noon ( $2 \mathrm{pm})$ and evening $(6 \mathrm{pm})]$ was used for the analysis. BL, at the start of treatment during a routine check-up; EL, at the end of 3-months of the treatment; TV, travoprost; LT, latanoprost.

The IOP is higher in the morning (23) and a previous cross-sectional study reported that $\mathrm{TV}$ was more effective in the management of IOP alone compared with LT (7) and with timolol (12); however, the present study was performed using an evening dose, as the higher IOP values in the morning may cause a type I error in the analysis (23). In addition, the manufacturers of TV (15) and LT (16) advise that the drops should be used in the evening in the management of glaucoma. The evening dose of prostaglandin analogues may provide exact effects to reduce the IOP.

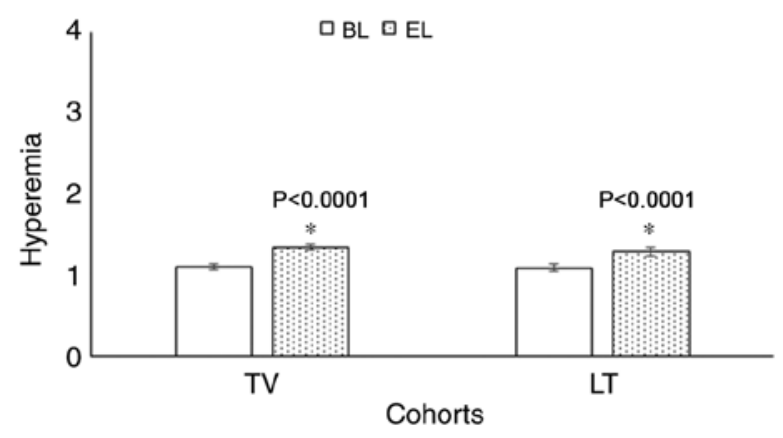

Figure 5. Hyperemia in the two groups at different time-points. Values (staining intensity score) are expressed as the mean \pm standard deviation. ${ }^{*} \mathrm{P}<0.05$ vs. BL. Staining was scored as follows: 0 , Absent; 1 , mild staining; 2 , moderate staining; and 3 , severe staining (maximum possible). BL, at the start of treatment during a routine check-up; EL, at the end of 3-months of the treatment; TV, travoprost; LT, latanoprost.

The present study suggested that an evening dose of TV was as safe as LT during the 3-months of treatment for patients with glaucoma, which was consistent with the results of a previous prospective nonrandomized study (1) and a retrospective multicenter review (24). This indicates that the prostaglandin analogs TV and LT may have a similar safety profile.

In the present study, a comparatively larger proportion of patients receiving TV treatment experienced dry eyes, while patients with LT treatment had more eyelid swelling and headache following 3-months of treatment, while there was 
Table II. Safety assessment.

\begin{tabular}{|c|c|c|c|c|c|c|}
\hline \multirow[b]{2}{*}{ Item } & \multicolumn{3}{|c|}{ TV group } & \multicolumn{3}{|c|}{ LT group } \\
\hline & $\mathrm{BL}(\mathrm{n}=89)$ & $\mathrm{EL}(\mathrm{n}=89)$ & P-value & $\mathrm{BL}(\mathrm{n}=161)$ & $\operatorname{EL}(n=161)$ & P-value \\
\hline Best-corrected visual acuity & $9.25 \pm 1.88$ & $9.24 \pm 1.81$ & 0.971 & $9.27 \pm 1.89$ & $9.26 \pm 1.88$ & 0.962 \\
\hline Visual field defect & $-0.66 \pm 0.09$ & $-0.67 \pm 0.10$ & 0.484 & $-0.65 \pm 0.08$ & $-0.66 \pm 0.09$ & 0.293 \\
\hline Vertical cup to disc ratio & $0.61 \pm 0.03$ & $0.62 \pm 0.04$ & 0.061 & $0.62 \pm 0.05$ & $0.63 \pm 0.06$ & 0.105 \\
\hline
\end{tabular}

Values are expressed as the mean \pm standard deviation. At present, there is no indication of a between-group comparison. The Wilcoxon test were performed for statistical analysis. $\mathrm{n}$ indicates the number of eyes. BL, at the start of treatment during a routine check-up; EL, at the end of 3 months of treatment; TV, travoprost; LT, latanoprost.

Table III. Qualitative results of the slit-lamp examination.

\begin{tabular}{|c|c|c|c|c|c|c|}
\hline \multirow[b]{2}{*}{ Location } & \multicolumn{3}{|c|}{ TV group } & \multicolumn{3}{|c|}{ LT group } \\
\hline & $\operatorname{BL}(\mathrm{n}=89)$ & $\mathrm{EL}(\mathrm{n}=89)$ & P-value & $\operatorname{BL}(n=161)$ & $\mathrm{EL}(\mathrm{n}=161)$ & P-value \\
\hline Eyelids & $1.03 \pm 0.02$ & $1.04 \pm 0.05$ & 0.082 & $1.04 \pm 0.04$ & $1.05 \pm 0.06$ & 0.079 \\
\hline Cornea & $1.02 \pm 0.03$ & $1.03 \pm 0.05$ & 0.108 & $1.01 \pm 0.03$ & $1.02 \pm 0.06$ & 0.060 \\
\hline Conjunctiva & $1.03 \pm 0.03$ & $1.04 \pm 0.04$ & 0.061 & $1.04 \pm 0.05$ & $1.05 \pm 0.09$ & 0.219 \\
\hline Iris & $1.09 \pm 0.05$ & $1.10 \pm 0.05$ & 0.184 & $1.08 \pm 0.04$ & $1.09 \pm 0.06$ & 0.079 \\
\hline Anterior chamber & $1.02 \pm 0.04$ & $1.03 \pm 0.05$ & 0.142 & $1.06 \pm 0.06$ & $1.07 \pm 0.07$ & 0.170 \\
\hline Lens & $1.60 \pm 0.05$ & $1.61 \pm 0.06$ & 0.229 & $1.58 \pm 0.09$ & $1.59 \pm 0.10$ & 0.346 \\
\hline
\end{tabular}

Values are expressed as the mean \pm standard deviation. 0 , Absent; 1 , mild; 2 , moderate; 3 , severe (maximum possible). At present, there is no indication of between-group comparison. The Wilcoxon test was performed for statistical analysis. $\mathrm{n}$ indicates the number of eyes. BL, at the start of treatment during a routine check-up; EL, at the end of 3-months of the treatment; TV, travoprost; LT, latanoprost.

Table IV. Treatment-emergent adverse effects after 3 months.

\begin{tabular}{|c|c|c|c|}
\hline Adverse effect & TV $(n=89)$ & LT $(n=161)$ & P-value \\
\hline Eye pain & $8(9)$ & 7 (4) & 0.167 \\
\hline Change in color of the iris & 4 (4) & $11 \quad(7)$ & 0.583 \\
\hline Eye discomfort & $12(13)$ & $15(9)$ & 0.192 \\
\hline Dry eye & $11(12)$ & $8(5)$ & $0.020^{\mathrm{a}}$ \\
\hline Itching in eye & 4 (4) & 7 (4) & 0.999 \\
\hline Irritation in eye & $5(6)$ & $16(10)$ & 0.341 \\
\hline Eyelid swelling & 1 (1) & $12(7)$ & $0.036^{\mathrm{b}}$ \\
\hline Skin rash & 1 (1) & $5 \quad(3)$ & 0.426 \\
\hline Headache & $2(2)$ & $15(9)$ & $0.037^{\mathrm{b}}$ \\
\hline
\end{tabular}

a Significant side effect of TV. ${ }^{\mathrm{b}}$ Significant side effect of LT. Values are expressed as n (\%). Fischer's exact test was performed for statistical analysis. $\mathrm{n}$ indicates the number of reported adverse-effect; $\mathrm{TV}$, travoprost; LT, latanoprost.

no difference in the frequency of discomfort, irritation and hyperemia between the groups. The results were consistent with those of a previous randomized controlled trial (8), an open-label, prospective, non-randomized cohort study (13), a double-blinded randomized trial (9), a single-center, open-label trial (14), and several randomized crossover studies $(12,17,20)$

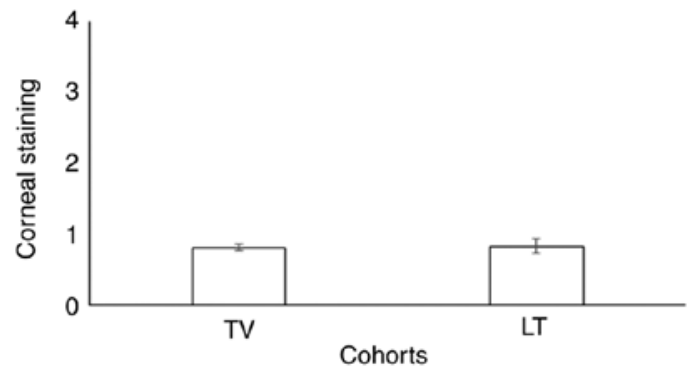

Figure 6. Results of corneal staining evaluation at the end of 3-months of treatment. Values (staining intensity score) are expressed as the mean \pm standard deviation. Staining was scored as follows: 0 , Absent; 1 , mild staining; 2 , moderate staining; and 3 , severe staining (maximum possible).

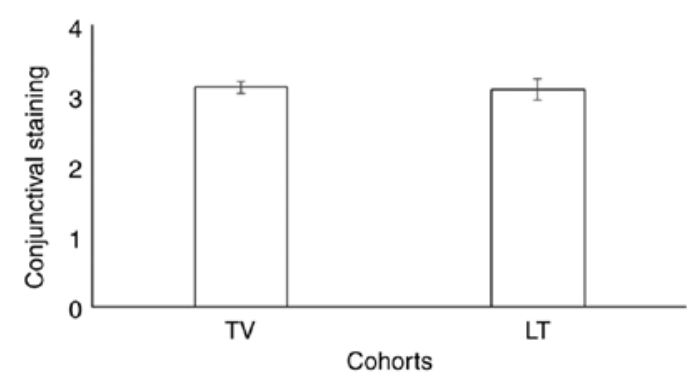

Figure 7. Results of conjunctival staining evaluation at the end of 3-months of treatment. Values are expressed as the mean \pm standard deviation. 0, Absent; 1, mild staining; 2 , moderate staining; and 3, severe staining (maximum possible). 
and prospective observational studies $(18,21)$. Of note, different prostaglandin analogs act on different inflammatory pathways (1). Long-term follow-up is required to evaluate the adverse effects of treatment with prostaglandin analogs in the management of glaucoma.

According to the manufacturer, Icare ${ }^{\circledast} \mathrm{PRO}$ is a high-precision tonometer; however, the present study used the Goldman Applanation Tonometer for measuring IOP. There is a lack of agreement regarding the accuracy of the Icare ${ }^{\circledR}$ PRO tonometer, while the reports for the Goldman Applanation Tonometer are consistent and provide an improved interpretation of the IOP-lowering effect of anti-glaucoma medication (25). Clinicians should be aware of the limitations of their labeled devices used in experimental studies.

The present study is novel, as it provided a justification for the use of the Goldman Applanation Tonometer; the results are in contrast to those of a previous study (as discussed above regarding the evening dose) and the present study provides evidence for the preferred use of an evening dose. However, there are several limitations. For instance, the present study was a retrospective study with a relatively short duration and lacking a control group. A longitudinal study is required, as different prostaglandin analogs have a different time-span for peak action (1). The follow-up period was relatively short and did not include the result of the visual field test of the patients. The interpretation of the IOP may be affected by the central corneal thickness; however, this was not considered in the present study.

In conclusion, the evening dose of TV and LT had the same efficacy and safety in the management of primary open-angle glaucoma with hyperemia (was significantly increased at 3 months vs. baseline, but there was no significant difference between the two drugs) and a relatively low frequency of manageable adverse effects following treatment. However, a large randomized trial is recommended to further support the present results.

\section{Acknowledgements}

Not applicable.

\section{Funding}

No funding received.

\section{Availability of data and materials}

The datasets used and analyzed during the current study available from the corresponding author on reasonable request.

\section{Authors' contributions}

All authors read and approved the study prior to submission for publication. JL, as the project administrator, contributed measurement of data and their evaluation and resources, and performed checking of the results or internal/external data/analysis and literature review of the study. XW contributed to the methodology, supervision, software and literature review of the study. GX contributed to the investigation, resources, formal analysis and literature review of the study.
RD contributed to formal analysis, data curation, resources and literature review of the study. LW contributed to supervision, data curation, validation and literature review of the study. LZ contributed to validation, supervision, software and literature review of the study. ZC contributed to software, formal analysis, data curation and literature review of the study and drafted, reviewed and edited the manuscript for intellectual content. The author agrees to be accountable for all aspects of work ensuring integrity and accuracy.

\section{Ethics approval and consent to participate}

The designed protocol (HMC/CL/14/19 dated 9 October 2019) of the present study was approved by the review board of Huizhou Municipal Central Hospital (Huizhou, China). The study adheres to the Law of China. Data of patients were collected from institute following written approval from Huizhou Municipal Central Hospital, China. The patients included provided written informed consent for treatment during hospitalization.

\section{Patient consent for publication}

Not applicable.

\section{Competing interests}

The authors declare that they have no competing interests.

\section{References}

1. Ayala $\mathrm{M}$ and Chen E: The influence of topical prostaglandin analogues in inflammation after selective laser trabeculoplasty treatment. J Ocul Pharmacol Ther 28: 118-122, 2012.

2. Zhang XL and Qin L: Efficacy of travoprost for the treatment of patients with glaucoma. Medicine (Baltimore) 98: e16526, 2019.

3. Stalmans I, Oddone F, Cordeiro MF, Hommer A, Montesano G, Ribeiro L, Sunaric-Mégevand G and Rossetti L: Comparison of preservative-free latanoprost and preservative-free bimatoprost in a multicenter, randomized, investigator-masked cross-over clinical trial, the SPORT trial. Graefes Arch Clin Exp Ophthalmol 254: 1151-1158, 2016.

4. Congdon N, O'Colmain B, Klaver CC, Klein R, Muñoz B, Friedman DS, Kempen J, Taylor HR and Mitchell P; Eye Diseases Prevalence Research Group: Causes and prevalence of visual impairment among adults in the United States. Arch Ophthalmol 122: 477-485, 2004.

5. Song P, Wang J, Bucan K, Theodoratou E, Rudan I and Chan KY: National and subnational prevalence and burden of glaucoma in China: A systematic analysis. J Glob Health 7: 020705, 2017.

6. Ernest PJ, Schouten JS, Beckers HJ, Hendrikse F, Prins MH and Webers CA: An evidence-based review of prognostic factors for glaucomatous visual field progression. Ophthalmology 120: 512-519, 2013.

7. Denis P, Launois R, Devaux M and Berdeaux G: Comparison of diurnal intraocular pressure control by latanoprost versus travoprost: Results of an observational survey. Clin Drug Investig 26: 703-714, 2006.

8. Garway-Heath DF, Crabb DP, Bunce C, Lascaratos G, Amalfitano F, Anand N, Azuara-Blanco A, Bourne RR, Broadway DC, Cunliffe IA, et al: Latanoprost for open-angle glaucoma (UKGTS): A randomised, multicentre, placebo-controlled trial. Lancet 385: 1295-1304, 2015.

9. Peace JH, Ahlberg P, Wagner M,Lim JM, Wirta D and Branch JD: Polyquaternium-1-preserved travoprost $0.003 \%$ or benzalkonium chloride-preserved travoprost $0.004 \%$ for glaucoma and ocular hypertension. Am J Ophthalmol 160: 266-274, 2015. 
10. Chander A, Kapoor $\mathrm{H}$ and Thomas S: Comparison of the efficacy and safety of bimatoprost $(0.03 \%)$ and travoprost $(0.004 \%)$ in patients with primary open angle glaucoma. Nepal $\mathbf{J}$ Ophthalmol 5: 75-80, 2013.

11. Tang W, Zhang F, Liu K and Duan X: Efficacy and safety of prostaglandin analogues in primary open-angle glaucoma or ocular hypertension patients: A meta-analysis. Medicine (Baltimore) 90: e16597, 2019.

12. Shoji T, Sato H, Mizukawa A, Hirota N, Enoki T, Kojima T, Kanda T and Takeuchi M: Hypotensive effect of latanoprost/timolol versus travoprost/timolol fixed combinations in NTG patients: A randomized, multicenter, crossover clinical trial. Invest Ophthalmol Vis Sci 54: 6242-6247, 2013.

13. Rahmatnejad K, Rapuano CJ, Ichhpujani P, Wizov SS, Moster MR, Hark LA and Katz LJ: The effects of latanoprost with benzalkonium chloride versus travoprost with SofZia on the ocular surface. Eye Contact Lens 44 (Suppl 2): S93-S98, 2018.

14. El Hajj Moussa WG, Farhat RG, Nehme JC, Sahyoun MA, Schakal AR, Jalkh AE, Abi Karam MP and Azar GG: Comparison of efficacy and ocular surface disease index score between bimatoprost, latanoprost, travoprost, and tafluprost in glaucoma patients. J Ophthalmol 2018: 1319628, 2018.

15. Alcon Laboratories Inc.: Package leaflet: Information for the user TRAVATAN ${ }^{\circledR} 40 \mathrm{micrograms} / \mathrm{ml}$ eye drops, solution travoprost. Available from: https://www.medicines.org. uk/emc/files/pil.1556.pdf. Accessed October 1, 2019.

16. Pfizer Inc.. Package leaflet: Information for the user Xalatan ${ }^{\circledR}$ $50 \mathrm{micrograms} / \mathrm{ml}$ eye drops, solution latanoprost. Available from: https://www.medicines.org.uk/emc/files/pil.1616.pdf. Accessed October 1, 2019.

17. Konstas AG, Kozobolis VP, Katsimpris IE, Boboridis K, Koukoula S, Jenkins JN and Stewart WC: Efficacy and safety of latanoprost versus travoprost in exfoliative glaucoma patients. Ophthalmology 114: 653-657, 2007.
18. Parmaksiz S, Yüksel N, Karabas VL, Ozkan B, Demirci G and Caglar Y: A comparison of travoprost, latanoprost, and the fixed combination of dorzolamide and timolol in patients with pseudoexfoliation glaucoma. Eur J Ophthalmol 16: 73-80, 2006.

19. Blondeau P, Hamid M and Ghalie Z: Prospective randomized clinical trial on the effects of latanoprost, travoprost and bimatoprost on latanoprost non-responders. J Fr Ophtalmol 42: 894-899, 2019.

20. Sawada A, Yamamoto T and Takatsuka N: Randomized crossover study of latanoprost and travoprost in eyes with open-angle glaucoma. Graefes Arch Clin Exp Ophthalmol 250: 123-129, 2012.

21. Mishra D, Sinha BP and Kumar MS: Comparing the efficacy of latanoprost $(0.005 \%)$, bimatoprost $(0.03 \%)$, travoprost $(0.004 \%)$, and timolol $(0.5 \%)$ in the treatment of primary open angle glaucoma. Korean J Ophthalmol 28: 399-407, 2014.

22. Setia MS: Methodology series module 3: Cross-sectional studies. Indian J Dermatol 61: 261-264, 2016.

23. Yan DB, Battista RA, Haidich AB and Konstas AG: Comparison of morning versus evening dosing and 24-h post-dose efficacy of travoprost compared with latanoprost in patients with open-angle glaucoma. Curr Med Res Opin 24: 3023-3027, 2008.

24. Fain JM, Kotak S, Mardekian J, Bacharach J, Edward DP, Rauchman S, Brevetti T, Fox JL and Lovelace C: A multicenter, retrospective chart review study comparing index therapy change rates in open-angle glaucoma or ocular hypertension patients newly treated with latanoprost or travoprost-Z monotherapy. BMC Ophthalmol 11: 13, 2011.

25. Rödter TH, Knippschild S, Baulig C and Krummenauer F: Meta-analysis of the concordance of Icare ${ }^{\circledR}$ PRO-based rebound and Goldmann applanation tonometry in glaucoma patients. Eur J Ophthalmol 30: 245-252, 2020. 